\title{
Comparison of Difference in Hematologic and Hemodynamic Outcomes between Primary Total Knee Arthroplasty and Revision of Infected Total Knee Arthroplasty
}

\author{
Oog-Jin Shon, MD, Dong-Chul Lee, MD, Seung Min Ryu, MD, and Hyo Sae Ahn, MD \\ Department of Orthopedic Surgery, Yeungnam University Medical Center, Daegu, Korea
}

Purpose: This study is to identify preoperative cautions for revision of infected total knee arthroplasty (TKA) by understanding the differences in hematologic and hemodynamic changes between primary TKA and revision of infected TKA.

Materials and Methods: The study included 40 patients in each of the two groups: one group with patients who underwent TKA and the other group with patients who underwent revision of infected TKA. All patients matched for age and body mass index. The following data were compared between the groups: changes in blood pressure, variations in hemoglobin level, amount of postoperative blood loss and transfused blood, incidence of blood transfusion, white blood cell (WBC) count, albumin level, erythrocyte sedimentation rate (ESR), C-reactive protein (CRP), and liver enzyme level.

Results: The hemoglobin levels, transfusion rate, and the amount of blood loss were significantly higher in the revision group ( $\mathrm{p}=0.012$ ). In both groups, CRP reached the highest level on the 3rd postoperative day but it was normalized 2 weeks postoperatively; however, the revision TKA group showed a greater tendency to normalization $(\mathrm{p}=0.029)$. There were significant differences between the groups in ESR, WBC, blood pressure, and changes in liver enzyme levels.

Conclusions: Revision of infected TKA results in greater hemodynamic variations than primary TKA. Therefore, more efforts should be made to identify pre- and postoperative hemodynamic changes and hematologic status.

Keywords: Knee, Arthroplasty, Infection, Revision, Hemodynamics, Hematology

\section{Introduction}

According to Tahmasebi et al. ${ }^{1)}$, total knee arthroplasty (TKA) can cause a lot of hematologic and hemodynamic burden on the human body due to intraoperative blood loss of about $265 \mathrm{~mL}$

Received September 13, 2015; Revised (1st) December 9, 2015;

(2nd) December 14, 2015; (3rd) January 27, 2016;

Accepted January 28, 2016

Correspondence to: Hyo Sae Ahn, MD

Department of Orthopedic Surgery, Yeungnam University Medical

Center, 170 Hyeonchung-ro, Nam-gu, Daegu 42415, Korea

Tel: +82-53-620-3640, Fax: +82-53-628-4020

E-mail: christiansio@hanmail.net

This is an Open Access article distributed under the terms of the Creative Commons Attribution Non-Commercial License (http://creativecommons.org/licenses/by-nc/4.0/) which permits unrestricted non-commercial use, distribution, and reproduction in any medium, provided the original work is properly cited. and postoperative blood loss of $554 \mathrm{~mL}$, reduction of hemoglobin $(\mathrm{Hb})$ level by $2.53 \mathrm{~g} / \mathrm{dL}$, and transfusion of 0.43 unit of packed red blood cells in addition to several postoperative complications ${ }^{2-6)}$.

In the study of Frisch et al. ${ }^{7}$, the incidence of deep infection after TKA was higher in the transfused group than the group without transfusion ( $2.4 \%$ vs. $0.5 \%)$; therefore, they suggested the surgeon be concerned about hemodynamic changes ${ }^{7,8)}$. Revision of infected TKA involves extensive soft tissue resection and bone cutting, resulting in more severe blood loss during and after surgery than primary TKA ${ }^{9,10}$. Accordingly, the risk of complications also increases after revision TKA.

Prasad et al. ${ }^{2)}$ described the transfusion rate of revision TKA and primary TKA as $19.1 \%$ and $14.8 \%$, respectively, indicating the rate was relatively higher in the revision of infected TKA. They also reported that the transfusion rate in primary TKA was 
associated with increased risk of postoperative mortality and deep infection. Therefore, it was considered that the revision group would more likely to have higher prevalence of complications.

Studies on the comparison of serological findings after TKA and revision of infected TKA have been rare. In this study, we analyzed the laboratory findings and compared the differences in hemodynamic and hematologic changes between the primary TKA group and revision of infected TKA group. The objective of this study was to identify preoperative cautions for revision of infected TKA by understanding the differences in hematologic and hemodynamic changes between the two groups.

\section{Materials and Methods}

Of the 45 patients who underwent revision due to infection after TKA at our hospital between January, 2010 and July, 2012, 40 patients who had no recurrence of infection and were available for more than 1-year follow-up were included in the test group. Of the 273 patients who underwent primary TKA, 40 patients who were available for more than 1-year follow-up were included in the control group. Exclusion criteria were simultaneous bilateral TKA, Hb level of $<10 \mathrm{~g} / \mathrm{dL}$, blood coagulation-related disorders, morbid obesity (body mass index [BMI], $\geq 30 \mathrm{~kg} / \mathrm{m}^{2}$ ), and infection (Table 1). In both groups, the total cases were females. Primary TKA patients in the control group were selected to be matched for age, BMI, and presence of diabetes with the patients who underwent revision of infected TKA.

The mean follow-up period was 27 months (range, 15 to 119 months) in patients with revision of infected TKA (revision group) and 17 months (range, 13 to 25 months) in patients with

Table 1. Demographics of Primary Total Knee Arthroplasty (TKA) Group and Revision TKA Group

\begin{tabular}{lccc}
\hline \multicolumn{1}{c}{ Variable } & Primary & $\begin{array}{c}\text { Infected } \\
\text { revision }\end{array}$ & p-value \\
\hline No. of cases & 40 & 40 & 0.023 \\
Follow-up (mo), mean (range) & $22(20-25)$ & $34(24-65)$ & 0.041 \\
Age (yr), mean (range) & $69.9(61-80)$ & $67.1(53-81)$ & 0.043 \\
Gender & Female & Female & 0.045 \\
Body mass index $\left(\mathrm{kg} / \mathrm{m}^{2}\right)$ & 27.1 & 26.9 & 0.035 \\
DM (pts/each group) & 7 & 8 & 0.075 \\
Anemia (hemoglobin<12 g/dL) & 4 & 8 & 0.041 \\
\hline
\end{tabular}

Exclusion criteria: bilateral TKA, anemia (hemoglobin $<10$ ), coagulation disorder, obesity (body mass index $>30$ ).

DM: diabetes mellitus, pts: patients. primary TKA (TKA group). The mean age was 67.1 years (range, 53 to 81 years) in the revision group and 69.9 years (range, 61 to 80 years) in the TKA group.

As for surgical procedures, after a median skin incision, a medial parapatellar approach was used on the articular capsule along the medial region of knee to expose the medial and lateral sides of the femoral condyles and tibial plateau. Then, soft tissue removal was conducted including medial soft tissue release. Meniscus and anterior cruciate ligament removal was followed by osteotomy on the proximal tibia and distal femur. Blood loss was minimized with thorough cauterization throughout the procedure, and care was taken not to cause excessive manipulation of soft tissues. No patellar resurfacing was performed in the entire cases, but patellar margin cauterization was conducted.

Revision of infected TKA was performed when there was no finding of clinical inflammation at 6-8 weeks after primary TKA and when the white blood cell (WBC) count was less than 5 on the high magnification view in a frozen section test performed using a tissue sample removed at the operating room. For intraoperative finding of severe bone defects, metal blocks or bone grafts were used. For severe medial and lateral instability, constrained implants were utilized. An extension stem was used when constrained implants were used and stability of the substitute was considered necessary. The extension stem was anchored with cement during a cement-retained procedure.

Intramedullary bone plug was inserted to reduce bleeding in both groups, and the suction drainage tube was removed 48 hours after surgery. Antibiotics were prescribed for five days. In the revision group, methicillin-resistance Staphylococcus aureus (MRSA) was identified in 4 patients and the antibiotics were used for 7 days (Table 2). From the 2nd postoperative day, low molecular weight heparin was administered to prevent deep vein throm-

Table 2. Identification of Organisms of Revision Total Knee Arthroplasty

\begin{tabular}{lc}
\hline \multicolumn{1}{c}{ Variable } & No. of patients \\
\hline Gram (+) & 4 \\
methicillin-resistant Staphylococcus aureus & 4 \\
methicillin-resistant Staphylococcus epidermidis & 3 \\
methicillin-sensitive Staphylococcus aureus & 2 \\
methicillin-sensitive Staphylococcus epidermidis & \\
Streptococcus pyogenes & \\
Streptococcus agalactiae & \\
Gram (-) & 1 \\
Enterococcus faecalis & 1 \\
Sphingomonas paucimobilis & \\
\hline
\end{tabular}




\section{Shon et al. Hematologic Comparison between Primary TKA and Revision of PJI}

bosis. From the 3rd postoperative day, early ambulation and continuous passive motion exercises were implemented. From the 5th postoperative day, ambulation exercises and passive exercise therapy were performed. In both groups, the following data were evaluated: changes in systolic and diastolic blood pressure, $\mathrm{Hb}$ variation, postoperative blood loss, incidence and amount of blood transfusion, WBC count, albumin level, erythrocyte sedimentation rate (ESR), C-reactive protein (CRP), and aspartate aminotransferase/alanine aminotransferase (AST/ALT). Blood transfusion was performed when the postoperative $\mathrm{Hb}$ level was $\leq 9 \mathrm{~g} / \mathrm{dL}$ and when it was reduced by $\geq 20 \%$ compared to the preoperative $\mathrm{Hb}_{\mathrm{b}}$ level ${ }^{11)}$. Statistical analysis was performed using SPSS ver. 18.0 (SPSS Inc., Chicago, IL, USA). For determination of statistical significance, a cross-analysis, correlation analysis, independent $t$-test, and repeated measures analysis of variance (especially all figures) were used.

\section{Results}

The $\mathrm{Hb}$ count was reduced on average by 1.1 (range, 0 to $2.9 \mathrm{~g}$ / $\mathrm{dL}$ ) in the TKA group and by 2.4 (range, 0.8 to $3.8 \mathrm{~g} / \mathrm{dL}$ ) in the revision group; the value was statistically significantly higher in the revision group. The $\mathrm{Hb}$ count returned to normal range at one week after surgery in both groups (Fig. 1).

As for the transfusion rate, a total of 5 subjects in the TKA group and a total of 8 subjects in the revision group received blood transfusion ( $12 \%$ vs. $20 \%$ ), showing statistically significant intergroup difference. The average amount of blood transfusion received was 0.5 (range, 0 to 2 pack) pack in the TKA group and 1.7 (range, 0 to 2 pack) packs in the revision group (Table 3 ).

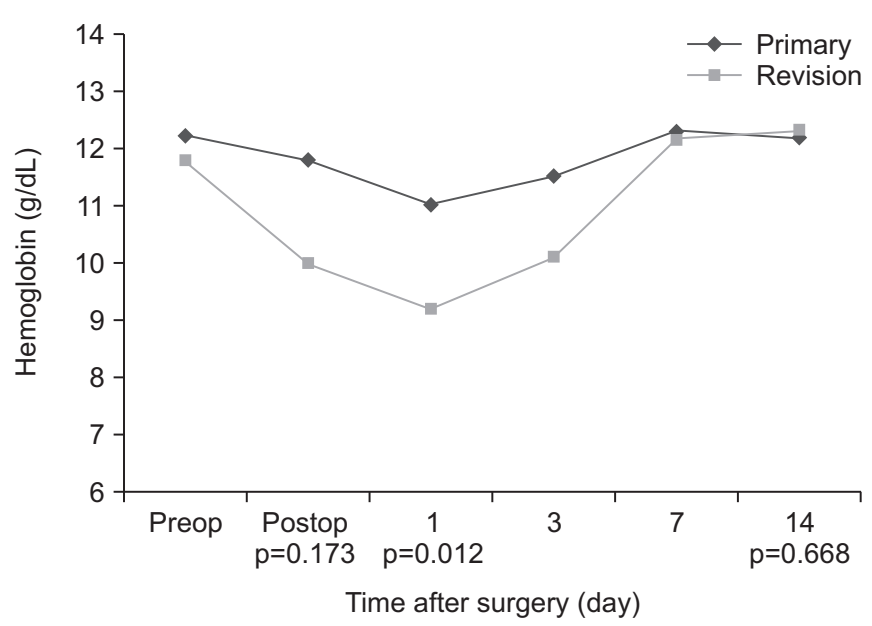

Fig. 1. Mean hemoglobin in patients who underwent primary total knee arthroplasty (TKA) or revision TKA. Preop: preoperative, Postop: postoperative.
Because it was not possible to compare the amount of blood loss during surgery, the amount of blood loss after surgery was compared instead. The relationship between the operation time and the amount of blood loss in use of hemovac was also assessed. The mean operation time was 58 minutes in the TKA group and 102 minutes in the revision group. The mean postoperative blood loss was $561 \mathrm{~mL}$ in the TKA group and $817 \mathrm{~mL}$ in the revision group. Therefore, the operation time and postoperative blood loss presented a statistically significant positive correlation (Table 4). The CRP level was assessed on the 3rd postoperative day and at each week from week 1 to week 6 after surgery. The highest CRP level was observed on the 3rd postoperative day in both groups: $9.1 \mathrm{mg} / \mathrm{dL}$ in the TKA group and $11.2 \mathrm{mg} / \mathrm{dL}$ in the revision group. The level was normalized in the 2 nd postoperative week, but it showed a tendency of being higher in the revision group, presenting a statistically significant intergroup difference (Fig. $2 \mathrm{~A})$. With regard to the ESR, the value was highest in the 2 nd postoperative week in both groups, but was normalized in the 6th postoperative week. On average, the value was higher in the revision group, but there were no statistically significant difference (Fig. 2B).

With regard to the WBC count, the value was highest on the 3rd postoperative day in both groups: $12,500 / \mu \mathrm{L}$ in the TKA group and $14,800 / \mu \mathrm{L}$ in the revision group. The value was normalized in the 2 nd postoperative week in both groups and there was no statistically significant intergroup difference (Fig. 3A).

Table 3. Comparison of Rate and Number of Transfusion

\begin{tabular}{lcc}
\hline Variable & $\begin{array}{c}\text { Need for } \\
\text { transfusion }(\%)\end{array}$ & $\begin{array}{c}\text { Packed red blood cell } \\
\text { transfusion (pack) }\end{array}$ \\
\hline Primary & $5(12)$ & $0.5 \pm 0.63$ \\
Revision & $8(20)$ & $1.7 \pm 0.54$ \\
p-value & 0.032 & 0.025 \\
\hline
\end{tabular}

Values are presented as mean \pm standard deviation or number.

Table 4. Comparison of Operation Time and Hemovac Volume after Operation between Primary Total Knee Arthroplasty (TKA) and Revision TKA

\begin{tabular}{cccc}
\hline Variable & $\begin{array}{c}\text { Operation time } \\
(\mathrm{min})\end{array}$ & $\begin{array}{c}\text { Hemovac volume } \\
(\mathrm{mL})\end{array}$ & p-value $^{\mathrm{a})}$ \\
\hline Primary & $58 \pm 3.38$ & $561 \pm 9.55$ & 0.012 \\
Revision & $102 \pm 5.17$ & $817 \pm 3.27$ & 0.023 \\
p-value $^{\mathrm{b})}$ & 0.027 & 0.025 & \\
\hline
\end{tabular}

Values are presented as mean \pm standard deviation.

${ }^{a}$ Correlation analysis (Pearson correlation coefficient: primary $=0.753$,

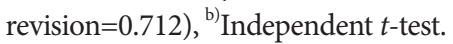


A

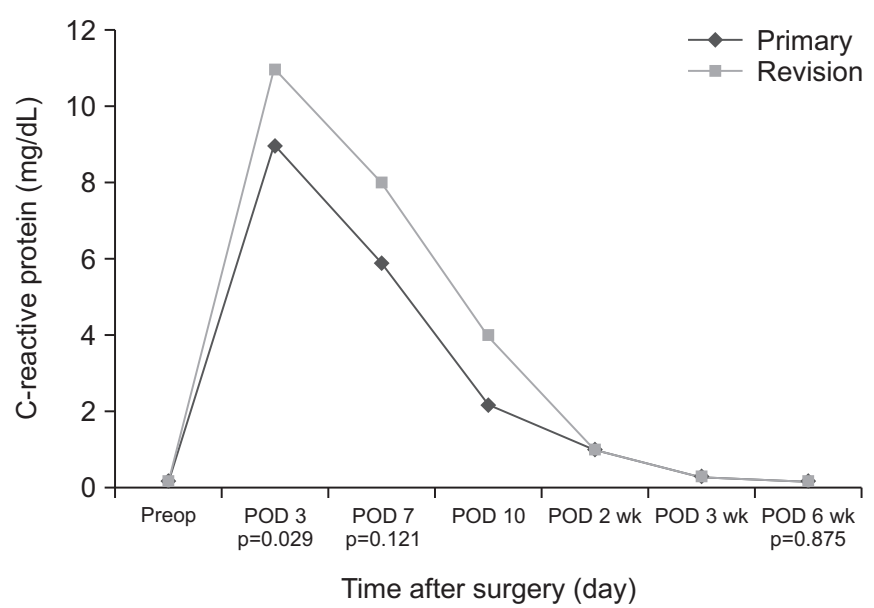

B

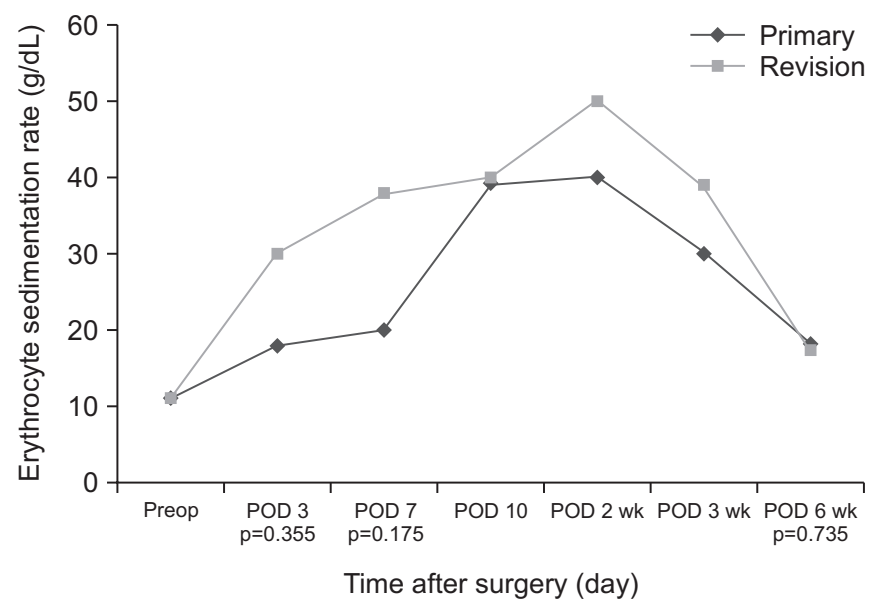

Fig. 2. (A) Mean plasma C-reactive protein concentrations in patients who underwent primary total knee arthroplasty (TKA) or revision TKA. (B) Mean erythrocyte sedimentation rates in patients who underwent primary TKA or revision TKA. Preop: preoperative, POD: postoperative day.

A

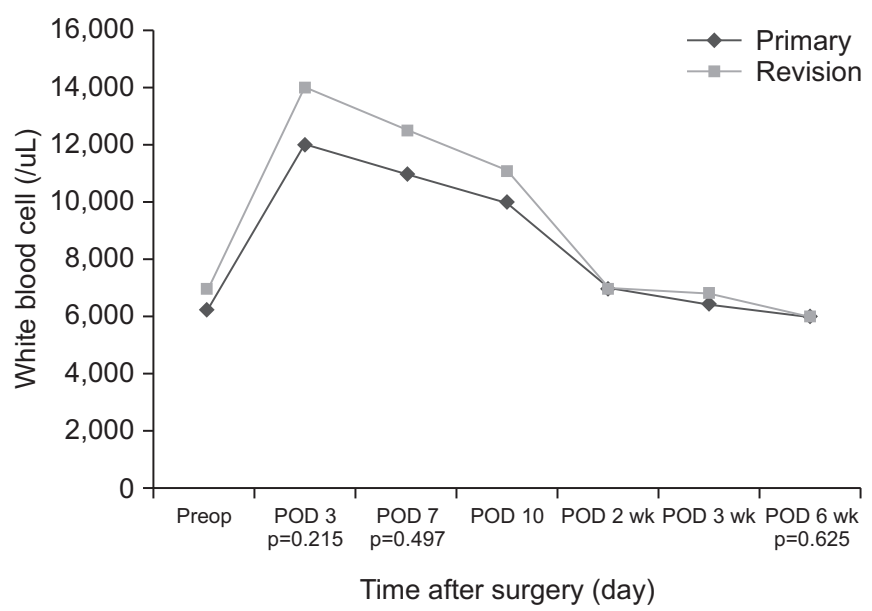

B

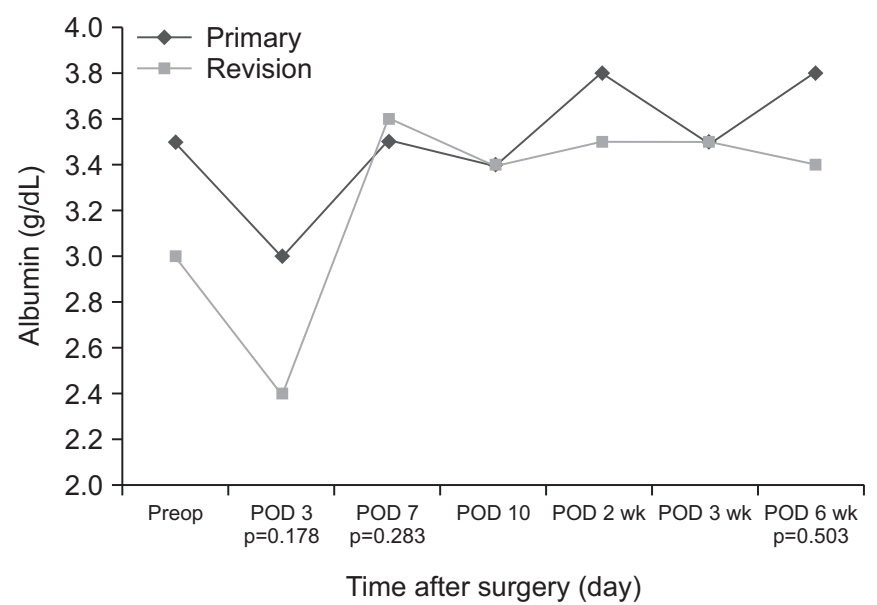

Fig. 3. (A) Mean white blood cell counts in patients who underwent primary total knee arthroplasty (TKA) or revision TKA. (B) Mean albumin level in patients who underwent primary TKA or revision TKA. Preop: preoperative, POD: postoperative day.

The albumin level was lowest on the 3rd postoperative day but was normalized in 1 week after surgery in both groups, and there was no statistically significant difference (Fig. 3B).

The postoperative systolic blood pressure slightly decreased immediately after surgery but was normalized in 12 hours after surgery in both groups (Fig. 4). The intraoperative variation and variation on the day of operation showed a positive input of body fluid causing a difference of about $200 \mathrm{~mL}$ in the revision group, but there was no statistically significant difference (Table 5).

In the revision group, the level of AST/ALT was elevated after preoperative intravenous infusion of antibiotics for about 5 weeks. Hepatotonics were administered to normalize the level and the surgery was performed after normalization. Elevation of the value was observed in 7 cases when antibiotics were used for over 7 days after surgery, and Hepatotonics were administered. There was no incidence of complications such as hepatic failure (Fig. 5).

\section{Discussion}

This study compared hemodynamic and hematologic changes between the TKA group and the revision group, and demonstrated significant differences between the two groups.

Tahmasebi et al. ${ }^{1)}$ and Aguilera et al. ${ }^{9)}$ reported that TKA causes a lot of changes to human body in terms of hematology and hemodynamics due to blood loss and the resulting reduction of $\mathrm{Hb}$ 


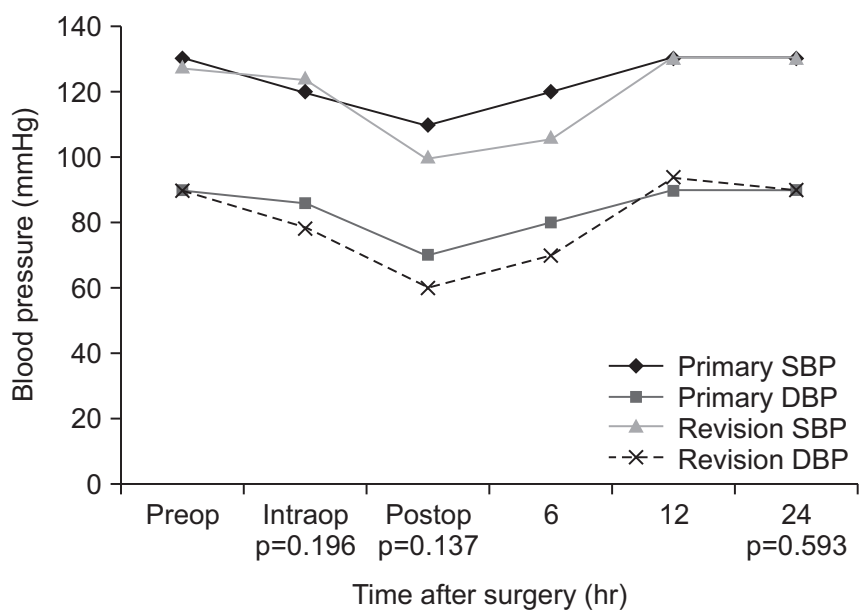

Fig. 4. Mean blood pressure level in patients who underwent primary total knee arthroplasty (TKA) or revision TKA. SBP: systolic blood pressure, DBP: diastolic blood pressure, Preop: preoperative, Intraop: intraoperative, Postop: postoperative.

Table 5. Comparison of Body Fluid between Intraoperative and Operation Day

\begin{tabular}{ccc}
\hline Variable & Intraoperative day $(\mathrm{mL})$ & Operation day $(\mathrm{mL})$ \\
\hline Primary & $250 \pm 8.8$ & $287 \pm 4.5$ \\
Revision & $430 \pm 9.8$ & $457 \pm 5.1$ \\
p-value & 0.23 & 0.17 \\
\hline
\end{tabular}

Values are presented as mean \pm standard deviation.

level and blood transfusion, which accordingly loads physical burden.

Prasad et al. ${ }^{2)}$ reported that the transfusion rate was higher with $19.1 \%$ in the revision group than $14.8 \%$ in the TKA group. Frisch et al. ${ }^{7)}$ and Hart et al. ${ }^{8)}$ reported that the high transfusion rate was associated with increased postoperative mortality and high risk of postoperative deep infection. In our study, the transfusion rate was higher in the revision group (20\%) than the TKA group (12\%); however, this finding should be confirmed in further studies because patients with infection were excluded from the analysis of transfusion rate.

Prasad et al. ${ }^{2)}$ reported that differences in hemodynamic and hematologic changes in the revision group could result from prolonged operation time, extensive incision range and larger amount of osteotomy, and manipulation of soft tissues. In our study, the postoperative blood loss, transfusion rate, and transfusion volume were greater in the revision group where the mean operation time was longer.

According to Aguilera et al. ${ }^{9}$, a revision for complicated infection involves greater intraoperative bleeding than primary TKA, and according to Samujh et al. ${ }^{3)}$, it can also result in greater post-

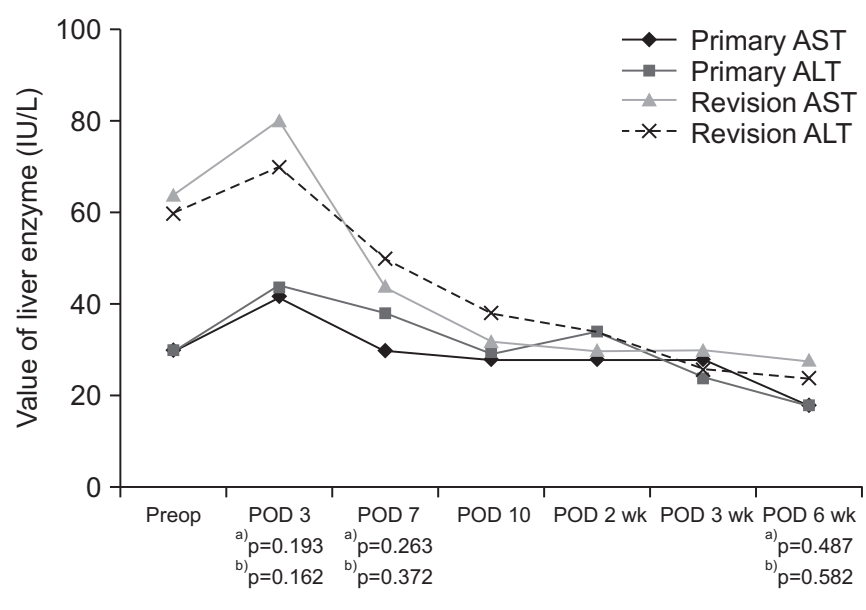

Time after surgery (day)

Fig. 5. Mean aspartate aminotransferase/alanine aminotransferase (AST/ ALT) level in patients who underwent primary total knee arthroplasty (TKA) or revision TKA. Preop: preoperative, POD: postoperative day. ${ }^{\text {a) }}$ AST, ${ }^{\text {b) }}$ ALT.

operative blood loss.

In the current study, the reduction of $\mathrm{Hb}$ count and the transfusion volume were greater in the revision group than the TKA group. When a large quantity of transfusion was required due to excessive bleeding, the group showed a tendency of having much lower systolic blood pressure.

Therefore, in revision of infected TKA, we suggest that the surgeon should pay more attention to the preoperative hemodynamic and hematologic conditions and prepare thorough measures in case of occurrence of postoperative complications.

There have been few studies on the relevance of liver function based on AST/ALT level and the long-term use of antibiotics. In the current study, we compared the AST/ALT level preoperatively and at week 1,2, and 6 after surgery in all patients. Although the number of patients with abnormally elevated levels of liver enzymes was 4 and 6, respectively, they were normalized on day 10 after surgery. There were some patients who presented with elevated levels, but normalization was confirmed after the use of Hepatotonics. In the revision group, such patients did not have complications such as fulminant hepatitis until up to about 14 days of its use.

The correlation between primary TKA and CRP level has been addressed in many studies ${ }^{12-16)}$. Bilgen et al. ${ }^{12)}$ reported that the level of CRP was elevated to the highest level on day 3 after surgery and was normalized on day 21 after surgery, which was not so different from the results of this study. However, there has been little research on the comparison of CRP level between the TKA group and the revision group. 
In case of CRP, statistically significant difference was observed at week 1 after surgery; however, no difference was observed in the mean value from the 2 nd postoperative week. Although 20 patients who underwent debridement and revision polyethylene implant arthroplasty were not included in this study due to postoperative acute infection, continuous elevation of CRP was observed in them from the 3rd postoperative week. Therefore, if continuous elevation of CRP is noted even after the 3rd postoperative week, acute infection should be suspected and total knee puncture should be performed.

In this study, MRSA was identified in 4 patients in the revision group; the incidence was relatively low compared to other studies $^{17,18)}$. The proper length of antibiotic treatment for MRSA in the revision group has not been established; however, based on empirical data, antibiotic treatment was performed 2 more days compared to the cases where no bacteria or a different strain was identified

This study has several limitations. Since it is a retrospective study, it could have resulted in biased sample selection. The power of the test for determining the adequate sample size was not conducted, which could have affected the outcomes of this study if the included number of samples was smaller than necessary. The number of subjects of this study was small. The followup period was short and there was no comparison with a group that underwent revision due to causes other than infection (aseptic loosening, etc.). In future studies, analyses of more variables should be conducted.

\section{Conclusions}

Postoperative changes in $\mathrm{Hb}$ level and CRP, transfusion rate and amount, and blood loss amount by postoperative Hemovac were greater at a statistically significant level in the revision group. Therefore, compared to primary TKA, in revision of infected TKA, more thorough efforts should be made to reduce postoperative complications by identifying preoperative hemodynamic changes and hematologic status and by analyzing and understanding the differences in instantaneous findings after surgery.

\section{Conflict of Interest}

No potential conflict of interest relevant to this article was reported.

\section{References}

1. Tahmasebi MN, Bashti K, Ghorbani G, Sobhan MR. Intraarticular administration of tranexamic acid following total knee arthroplasty: a case-control study. Arch Bone Jt Surg. 2014;2:141-5.

2. Prasad N, Padmanabhan V, Mullaji A. Blood loss in total knee arthroplasty: an analysis of risk factors. Int Orthop. 2007;31:39-44.

3. Samujh C, Falls TD, Wessel R, Smith L, Malkani AL. Decreased blood transfusion following revision total knee arthroplasty using tranexamic acid. J Arthroplasty. 2014;29(9 Suppl):182-5.

4. Spahn DR. Anemia and patient blood management in hip and knee surgery: a systematic review of the literature. Anesthesiology. 2010;113:482-95.

5. Levine BR, Haughom B, Strong B, Hellman M, Frank RM. Blood management strategies for total knee arthroplasty. J Am Acad Orthop Surg. 2014;22:361-71.

6. Namba RS, Inacio MC, Paxton EW. Risk factors associated with deep surgical site infections after primary total knee arthroplasty: an analysis of 56,216 knees. J Bone Joint Surg Am. 2013;95:775-82.

7. Frisch NB, Wessell NM, Charters MA, Yu S, Jeffries JJ, Silverton $\mathrm{CD}$. Predictors and complications of blood transfusion in total hip and knee arthroplasty. J Arthroplasty. 2014; 29(9 Suppl):189-92.

8. Hart A, Khalil JA, Carli A, Huk O, Zukor D, Antoniou J. Blood transfusion in primary total hip and knee arthroplasty. Incidence, risk factors, and thirty-day complication rates. J Bone Joint Surg Am. 2014;96:1945-51.

9. Aguilera X, Videla S, Almenara M, Fernandez JA, Gich I, Celaya F. Effectiveness of tranexamic acid in revision total knee arthroplasty. Acta Orthop Belg. 2012;78:68-74.

10. Huff TW, Sculco TP. Management of bone loss in revision total knee arthroplasty. J Arthroplasty. 2007;22(7 Suppl 3): 32-6.

11. Tavares Cardozo R, Fidelis de Souza Junior E, Campoli Alves W, Barbi Filho F. Total knee arthroplasty: indication of blood transfusion according to hematimetric variation and clinical symptoms of hypoperfusion. Rev Bras Ortop. 2014;49:507-12.

12. Bilgen O, Atici T, Durak K, Karaeminogullari, Bilgen MS. Creactive protein values and erythrocyte sedimentation rates after total hip and total knee arthroplasty. J Int Med Res. 2001;29:7-12.

13. Shih LY, Wu JJ, Yang DJ. Erythrocyte sedimentation rate and 


\section{Shon et al. Hematologic Comparison between Primary TKA and Revision of PJI}

C-reactive protein values in patients with total hip arthroplasty. Clin Orthop Relat Res. 1987;(225):238-46.

14. Carlsson AS. Erythrocyte sedimentation rate in infected and non-infected total-hip arthroplasties. Acta Orthop Scand. 1978;49:287-90.

15. Niskanen RO, Korkala O, Pammo H. Serum C-reactive protein levels after total hip and knee arthroplasty. J Bone Joint Surg Br. 1996;78:431-3.

16. Okafor B, MacLellan G. Postoperative changes of erythrocyte sedimentation rate, plasma viscosity and C-reactive pro- tein levels after hip surgery. Acta Orthop Belg. 1998;64:52-6.

17. Siddiqui MM, Lo NN, Ab Rahman S, Chin PL, Chia SL, Yeo SJ. Two-year outcome of early deep MRSA infections after primary total knee arthroplasty: a joint registry review. J Arthroplasty. 2013;28:44-8.

18. Parvizi J, Azzam K, Ghanem E, Austin MS, Rothman RH. Periprosthetic infection due to resistant staphylococci: serious problems on the horizon. Clin Orthop Relat Res. 2009; 467:1732-9. 